\title{
AVALIAÇÃO GEOESTATÍSTICA DA RELAÇÃO ENTRE O POTENCIAL DE DISSECAÇÃO DO RELEVO E A GEODIVERSIDADE DA REGIÃO CÁRSTICA DE ARCOS-PAINS-DORESÓPOLIS-IGUATAMA
}

\author{
Fabiano Érico de Souza ${ }^{1}$
}

Carlos $\mathrm{Lobo}^{2}$

\section{Resumo}

A quantificação de feições abióticas é um importante processo nos estudos focados em geodiversidade, uma vez que sua aplicação permite indicar e auxiliar na análise de relevância de áreas prioritárias para a geoconservação. Apesar da geodiversidade ser uma temática incipiente nas geociências, alguns métodos de quantificação são bem difundidos, sendo um deles, a equação proposta por Serrano e RuizFlanõ (2007). No entanto, ainda existem dúvidas perante sua aplicabilidade, principalmente no que se refere ao impasse entre sua fundamentação teórica e análise estatística da correlação entre os coeficientes da equação, no caso, a relação entre os coeficientes de geodiversidade e rugosidade. Nessa ótica, este estudo propõe analisar essa relação, por meio do modelo geoestatístico GeographicallyWeightedRegression (GWR), aplicado em sub-bacias da Região Cárstica de Arcos-Pains-Doresópolis-Iguatama. Para simular o coeficiente de rugosidade foi utilizado o Índice de Global de Dissecação do Relevo, o qual consiste em uma combinação de índices morfométricos que permitem estimar o poder de dissecação que uma determinada região possui. Como resultado, o modelo não conseguiu explicar sozinho essa relação de em escala regional, porém possibilitou a análise local de comportamentos distintos, através da espacialização do $\mathrm{R}^{2}$ local e dos resíduos.

Palavras-Chave:Geodiversidade, geoestatístico, quantificação

\begin{abstract}
The abiotic features quantification is an important process to Geodiversity focused studies, since its application allows indicating and assisting relevance analysis in priority areas to geoconservation. Although geodiversity is an incipient theme in geosciences, some qualification methods are well known, being one of them the equation proposed by Serrano and Ruiz-Flanõ (2007). However, there are some doubts regarding its applicability, mainly due to its theoretical basis and coefficients statistics correlation, in case the relation between geodiversity and roughness coefficients. In this perspective, this study proposes an analysis, by means of the geostatistical model, Geographically Weighted Regression (GWR), applied in sub-basins of the Arcos-Dores-Doresópolis-Iguatama Karst Region. In order to simulate roughness coefficient, Relief Global Index of Dissection is used, which consists of a combination of morphometric indices allowing to estimate the given region's dissecting power. As a result, the model was unable to explain this regional scale relationship alone, but it enabled a local analysis of distinct behaviors through local R2 and residues spatialization
\end{abstract}

Key-words:Geodiversity, geostatistics, quantification

\footnotetext{
${ }^{1}$ (UFMG - Departamento de Cartografia, Mestrando), fabianoerico@hotmail.com

${ }^{2}$ (UFMG - Departamento de Geografia, Professor), carlosfflobo@gmail.com
} 


\section{1- INTRODUÇÃO}

A Geodiversidade pode ser conceituada como uma característica de uma determinada paisagem, marcada pela heterogeneidade de elementos e formas do meio físico. Em outras palavras, é a diversidade de propriedades geológicas e geomorfológicas da superfície terrestre, a qual inclui estruturas e processos do relevo, litologia, diversidade de minerais, solos, sistemas e relações entre estes elementos (GRAY, 2004, p. 17).

Estudos com o enfoque na geodiversidade buscam apresentar e até quantificar arelevância dos elementos físicos no funcionamento dos sistemas ambientais. A combinação desses elementos podem ser essenciais para o funcionamento de um sistema ambiental, que em seu pleno equilíbrio, proporciona diversos serviços primordiais para a sociedade, como a manutenção de aquíferos e mananciais, controle de processos erosivos e até mesmo a formação de paisagens utilizadas para lazer e contemplação.

Nesse contexto, a quantificação da geodiversidade possui um importante papel, uma vez que proporciona suporte técnico para o reconhecimento de áreas de alta relevância e gestão da geoconservação. Com isso, o intuito principal da quantificação é definir o grau de relevância da geodiversidade de um determinado lugar, assim como, definir sua abrangência no espaço para propor intervenções objetivas (PEREIRA et al. 2013, p. 1).

Diante do exposto, este estudo visa quantificar a geodiversidade da Região Cárstica de Arcos-Pains-Doresópolis-Iguatama, fundamentando na equação proposta por Serrano e RuizFlanõ (2007) e analisar, por meio de um modelo geoestatístico, a relação do potencial de dissecação do relevo com o aumento da geodiversidade. Para isso, será empregado o Índice de Global de Dissecação do Relevo. Presume-se que um alto poder de dissecação, na qual a energia cinética da água é o agente modelador, condiciona uma maior variedade de formas estruturais, feições e processos.

\section{2- ÍNDICE DE GEODIVERSIDADE E O ÍNDICE DE POTENCIALIDADE DE DISSECAÇÃO DO RELEVO}

Uma metodologia bastante difundida para o cálculo da geodiversidade é a proposta por Serrano e Ruiz-Flanõ (2007), aplicada em TiermesCaracena, na Espanha. O índice gerado consiste no somatório de elementos físicos presentes em uma determinada área (geológicos, geomorfológicos, hidrológicos e pedológicos) multiplicado pela rugosidade do relevo. No entanto, 
o emprego do coeficiente de rugosidade1 é uma discussão presente em trabalhos espelhados em Serrano e Ruiz-Flanõ (2007), por apresentar, estatisticamente, baixa correlação com a geodiversidade (HYORT; LOUTO,2010, p. 114; PELLITERO,2012, p. 887). A expressão original do índice é dada a seguir:

\title{
$\mathbf{G d}=\mathbf{E g} R / \ln \mathrm{S}$
}

Onde: $\mathrm{Gd}=$ índice de geodiversidade; $\mathrm{Eg}=$ número de elementos presentes; $\mathrm{R}=$ coeficiente de rugosidade; $\ln =$ logaritmo Neperiano; $\mathrm{S}=$ área da superfície.

Para Serrano e Ruiz-Flanõ (2007) a utilização da rugosidade em seu índice é justificada pela seguinte forma:

\begin{abstract}
"La topografía y lasvariaciones microclimáticas y topoclimáticasestán representadas a través del coeficiente de rugosidad. Suinclusión se justifica por el papel de estosenlaorganización de losflujos de energía (insolación, humedad) y materiales (agua, sedimentos) enlasladeras y, enconsecuencia, enladiversidad y distribución de formas y procesos. Se trata de introducirunparámetro integrador que permita tenerencuentavariaciones menores y relaciones complejas entre los elementos del sistema natural abiótico" (SERRANO; RUIZ-FLANÕ, 2007, p. 87).
\end{abstract}

Ao embasarmos nos sistemas evolutivos clássicos de Davis (1899), Penck (1924),posteriormente King (1955) e Hack (1960), entende-se que os processos de denudação (processos erosivos), são os processos exógenos mais importantes para a morfogênese. Obviamente, existem outros fatores que controlam a intensidade dos processos erosivos: intensidade pluviométrica, características geológicas e litológicas, propriedades do solo, dentre outros. No entanto, a função de um modelo é realizar uma representação simplificada da realidade, sendo válida a tentativa de considerar apenas os pontos mais importantes de um determinado processo e atenuar fatores menos relevantes (CHRISTOFOLETT, 1999; CASSETI, 2005).

Em relação ao emprego de índices morfométrios, salienta-se não é algo recente esurge da necessidade de uma aplicação prática nos estudos hidrológicos em bacias hidrográficas. (HORTON, CHORLEY, 1962; CHORLEY e KENNEDY, 1971;CHRISTOFOLETTI, 1980; HACK, 1973; apud SOUZA et al. 2017, p. 3).

Embasados em índices conhecidos e com auxílio de ferramentas degeoprocessamento, Souza et al. (2017) propuseram o índice Global de Dissecação do Relevo - IGDR, através da junção de três índices geomorfológicos já consolidados na literatura: o Índice de Concentração da Rugosidade - ICR, desenvolvido por Sampaio (2008), Índice de Hack (StreamLength-Gradient

\footnotetext{
${ }^{1}$ Para Hobson (1972), a rugosidade corresponde a variação da declividade, podendo ser interpretada pela relação entre a área da superfície inclinada e a área da superfície planimétrica. Dessa forma: Rugosidade $(\mathrm{R})=(\mathrm{Si}) /(\mathrm{Sp})$
} 
Index),concebido por Hack (1973) e a Densidade de Drenagem, definida inicialmente dor Horton (1945). Desse modo, o índice permite a delimitação de compartimentos de relevo considerando adensidade de drenagem, a rugosidade do relevo e o vigor energético dos canais de drenagem, estimado pela relação declividade-extensão.

\section{3- ÁREA DE ESTUDO}

A área de estudo contempla o polígono denominado de Região Cárstica de ArcosPainsDoresópolis-Iguatama pelo Projeto Arcos Pains Espeleologia - PROAPE. O polígono possui cerca de $1.152 \mathrm{~km}^{2}$ e se encontra na Mesorregião do Oeste de Minas, englobando os municípios de Bambuí, Piumhi, Doresópolis, Pains, Pimenta, Córrego Fundo, Arcos, Iguatama e Formiga.

A região é reconhecida por ser um importante polo mínero-industrial devido aocorrência de espessos estratos de rochas calcárias do Grupo Bambuí que servem de matériaprima na produção de cimento, cal, corretivo de solo, dentre outros produtos. Também é reconhecida pelas belas paisagens e importantes acervos arqueológicos e paleontológicos reconhecidos nacionalmente.

No contexto geofisiográfico, a área de estudo se encontra na porção sul do Crátondo São Francisco, também na porção sul do Grupo Bambuí. A estratigrafia é basicamente constituída por rochas pelítica na base e rochas carbonatadas no topo. A área de interesse também abrange rochas metamórficas da Serra de Pimenta, localizada na porção sul/sudoeste do polígono (PROAPE, 2012, p. 19).

\section{4- MATERIAIS E MÉTODOS}

O processo metodológico de aquisição da geodiversidade foi realizado de formaindependente, levando em consideração as delimitações das sub-bacias existentes na área de estudo (base cartográfica IGAM, 2010). Como justificativa, a utilização de sub-bacias como unidade espacial permite análises isoladas, particularizando cada situação, sobretudo por serem consideradas unidades sistêmicas compostas por outros próprios subsistemas. Ao todo, foram consideradas 1.921 bacias, sendo estas, pertencentes às bacias do Rio Grande e Rio São Francisco.

A escolha das variáveis abióticas (Tabela 1) foi embasada nos dados utilizados em Pereira et al. (2013) e no conceito de geodiversidade proposto por Gray (2004), em que leva-se em consideração formas e processos abióticos. Os dados foram manipulados em formato vetorial e em estruturas distintas (ponto, linha, polígono). As bases digitais advém de mapeamentos secundários, 
bases cartográficas disponibilizadas por órgãos ambientais e de dados extraídos de subprodutos gerados a partir de imagenShuttle Radar TopographyMission - SRTM/NASA (folha 21S046W resolução espacial de 30m), mais precisamente, dados de declividade e curvaturas vertical e horizontal.

Tabela 1 - Variáveis utilizadas no cálculo da Geodivesidade

\begin{tabular}{|c|c|c|c|c|}
\hline Variáveis & Taxa & Formato & Fonte & Escala \\
\hline Afloramentos & $\begin{array}{c}\text { Área / área bacia } \\
\text { Quantidade/área bacia }\end{array}$ & $\begin{array}{c}\text { Polígono } \\
\text { Ponto }\end{array}$ & $\begin{array}{c}\text { MARTINS } \\
\text { (2013) } \\
\text { CPRM (2005) }\end{array}$ & $1: 150.000$ \\
\hline Cavidades & Quantidade/área bacia & Ponto & PROAPE (2012) & $1: 10.000$ \\
\hline Surgências/Sumidouros & Quantidade/área bacia & Ponto & PROAPE (2012) & $1: 10.000$ \\
\hline $\begin{array}{c}\text { Lineamentos/ Estruturas } \\
\text { Feições }\end{array}$ & Extensão/área bacia & Linha & $\begin{array}{c}\text { PROAPE (2012) } \\
\text { MARTINS } \\
(2013)\end{array}$ & $1: 150.000$ \\
\hline Litologia & Quantidade/área bacia & Polígono & $\begin{array}{c}\text { MARTINS } \\
(2013)\end{array}$ & $1: 150.000$ \\
\hline $\begin{array}{c}\text { CPRM (2005) } \\
\text { Anidades geomorfológicas }\end{array}$ & Área / área bacia & Polígono & $\begin{array}{c}\text { MARTINS } \\
(2013)\end{array}$ & $1: 150.000$ \\
\hline Rede de drenagem & Extensão/área bacia & Linha & MDE & $30 \mathrm{~m}$ \\
\hline $\begin{array}{c}\text { Processos erosivos } \\
\text { recentes }\end{array}$ & Área / área bacia & Polígono & MDE & $30 \mathrm{~m}$ \\
\hline $\begin{array}{c}\text { Processos deposicionais } \\
\text { recentes }\end{array}$ & Área / área bacia & Polígono & MDE & $30 \mathrm{~m}$ \\
\hline
\end{tabular}

Em virtude da estrutura dos dados e da diferença de tamanho das sub-bacias, foramcriados parâmetros normalizados para cada variável, definidos pela relação entre número de ocorrências existentes, extensão/comprimento ou área da feição dividido pela área total da bacia. Este processo foi realizado por meio de técnicas de geoprocessamento, com a utilização das ferramentas Intersecte Dissolve, disponíveis no ArcMap/Arcigis10.3, em que as informações geométricas das variáveis são indexadas ao polígono da bacia. Por fim, o índice de geodiversidade de cada bacia foi composto pela média aritmética simples dos índices das variáveis. As feições possuem o mesmo grau de importância por se tratar de uma metodologia com viés apenas quantitativo. Salienta-se que a utilização desses parâmetros evita a supervalorização de bacias de maior tamanho. Além disso, permite a comparação e relação entre variáveis de diferentes grandezas. 
Todo o processo de obtenção do Índice Global de Dissecação do Relevo - IGDR,bem como, as configurações das ferramentas utilizadas foram embasados em Souza et al. (2017). Os procedimentos são realizados no ambiente ArcMap/Arcigis10.3, com o auxílio das ferramentas KernelDensity e o interpolador InverseDistanceWeighted. Os dados topográficos usados nesta etapa também formam extraídos das imagem SRTM, mencionadas anteriormente.

A expressão utilizada para o cálculo do IGDR é descrita a seguir, a qual correspondeuma álgebra matricial por média ponderada cujas variáveis possuem o mesmo peso:

$$
\mathrm{IGDR}=(\mathrm{ICR} \text { Global } \times 0,33)+(\mathrm{IH} \times 0,33)+(\text { Den. } \text { Dre } \times 0,33)
$$

Os procedimentos metodológicos para a aquisição do ICR e do índice de Hack estãoresumidos nas Figuras 1 e 2.

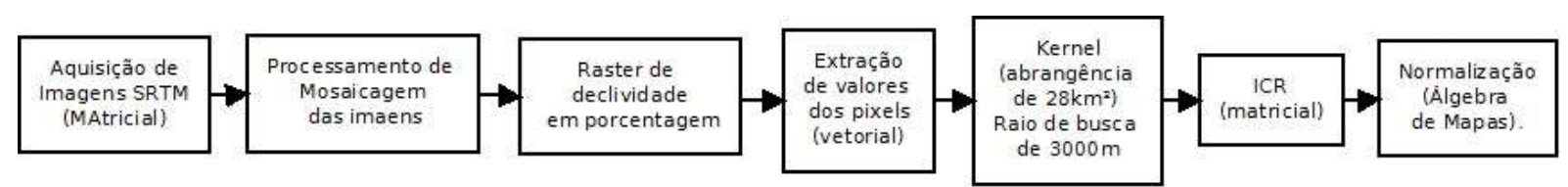

Figura 1 - Fluxograma de obtenção do Índice de Concentração da Rugosidade

Fonte: Souza et al. (2017)

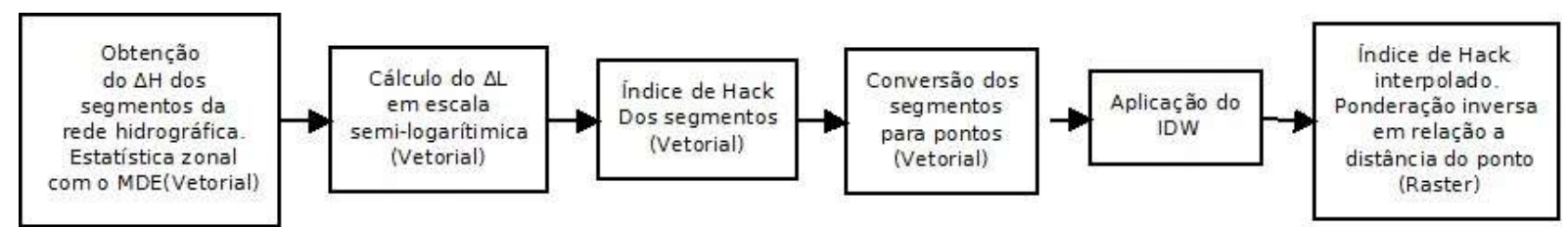

Figura 2 - Fluxograma da obtenção do Índice de Hack

Fonte: Souza et al. (2017)

A atribuição dos valores do IGDR para as bacias foi realizada por meio daferramenta Zonal Statistics as Table, onde foi retirada a média dos valores dos pixels que situam-se dentro dos polígonos das bacias.

Por fim, para avaliar a predição da geodiversidade (variável dependente) em funçãoda rugosidade (variável independente), representada pelo IGDR, foi utilizado o modelo GeographicallyWeightedRegression (GWR). Este modelo consiste em um método de regressão linear, aplicado espacialmente em nível local, considerando um raio de abrangência ou largura de banda para ponderar as observações, sendo preestabelecido pelo método AkaikeInformationCriterion (AICC). Por sua vez, o método AICC se adequa ao estudo proposto, no caso, a análise isolada das bacias. Conforme menciona Lobo et al. (2015), o método permite 
comparar diferentes modelos de regressão, o que possibilita melhor ajustamento dos dados observados. Como resultado, o modelo gera dados estatísticos, dentre eles, o índice de regressão local (R2) e o resíduo padronizado, sendo estes utilizados nas seguintes análises.

\section{5- $\quad$ ANÁLISE E DISCUSSÃO DOS RESULTADOS}

Pode-se relacionar o resultado da quantificação da geodiversidade (Figura 3)levando em consideração os dois sistemas distintos existentes na áreas de estudo: o sistema padrão, constituído por demais processos físicos e geoquímicos e o sistema cárstico, onde a rocha executa papel preponderante para o desenvolvimento das feições por dissolução.

Nessa ótica, destacam-se no sistema cárstico as sub-bacias próximas aos principaiscursos d'água, sobretudo nas proximidades do Rio São Miguel, onde existem um agrupamento significativo de bacias com alto valor de geodiversidade. Essa região possui alta concentração de afloramentos carbonáticos e cavidades naturais subterrâneas e, conforme estudos do PROAPE (2012), é a porção onde as rochas carbonáticas estão mais aflorantes. Também estão presentes processos de deposição aluvial mais recentes.

A porção sul/sudeoeste da área de estudo também se destaca pelos altos valoresde geodiversidade, sendo esta região caraterizada pela Serra de Piumhi, sustentada por rochas ígneas e metamórficas e onde concentra-se estruturas e processos erosivos mais ativos. Destacase também a grande concentração de bacias com geodiversidade muito baixa no centro e no extremo oeste da área de estudo. Apesar de muitas bacias estarem associadas ao domínio cárstico, nessas regiões predominam deposições sedimentares.

A aplicação do índice IGDR (Figura 4) evidenciou maior atividade de denudaçãona região da Serra de Piumhi, onde, de fato, o relevo é mais acidentado e possui maior densidade de cabeceiras de drenagem. As sub-bacias próximas ao contato entre dois sistemas, bem como as localizadas nas cabeceiras dos principais rios também se destacam pelos altos valores. A noroeste, os altos valores do índice podem estar vinculados ao relevo acidentado, proporcionado pelos cânions do Rio São Francisco. Os baixos valores estão relacionados as áreas de deposição e baixa densidade de drenagem.

A regressão linear simples, utilizando $95 \%$ de nível de confiança, demonstrou queas variáveis estão correlacionadas positivamente. Entretanto, o R2 ajustado foi muito baixo $(0,05)$, remetendo a sua baixa capacidade de predição entre a geodiversidade e o IGDR. Logo, este 
modelo não é o mais adequado para a análise aqui proposta, principalmente por se tratar de uma relação entre variáveis espaciais.

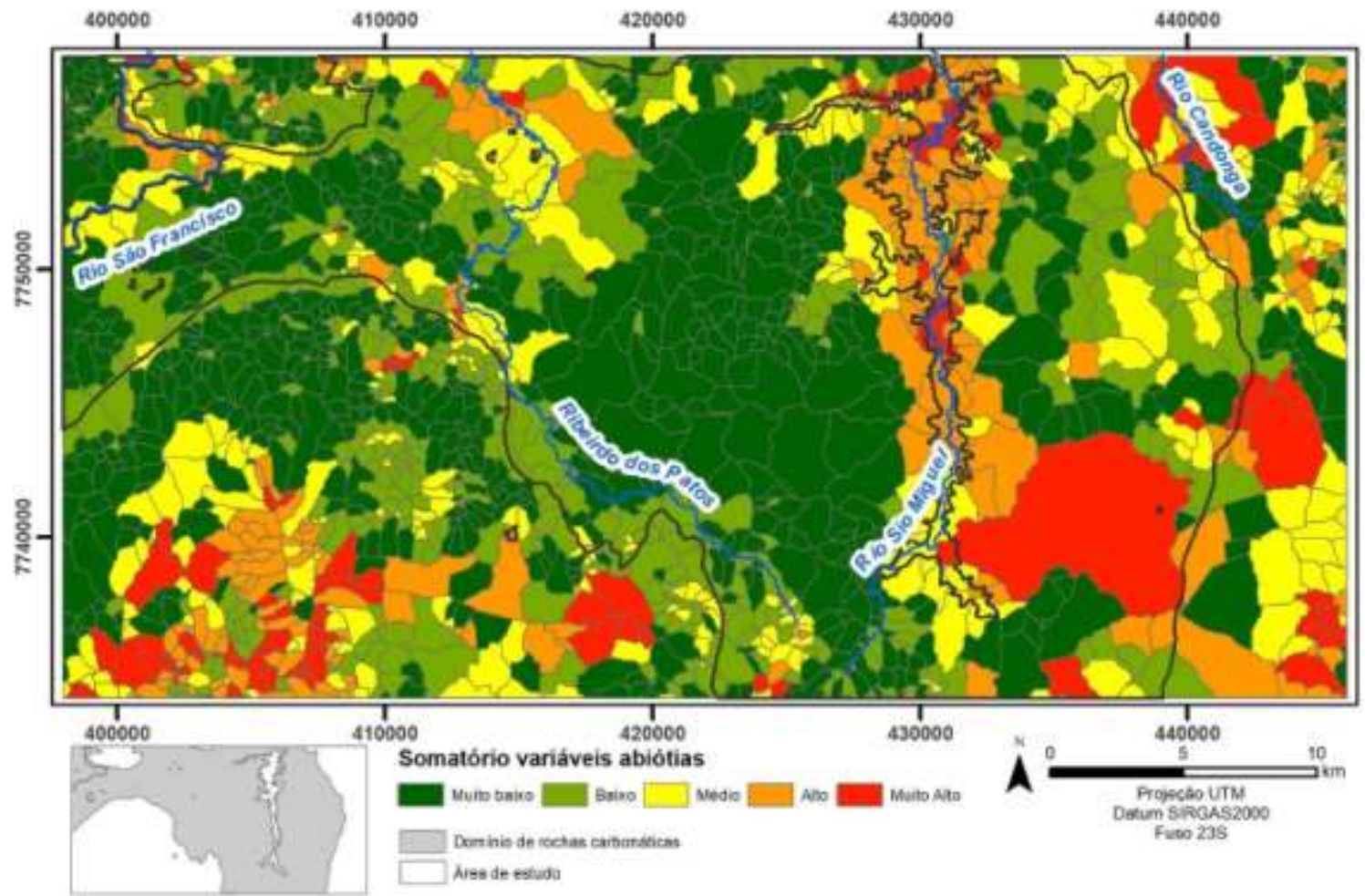

Figura 3 - Índice de geodiversidade daRegião Cárstica de Arcos-Pains-Doresópolis-Iguatama

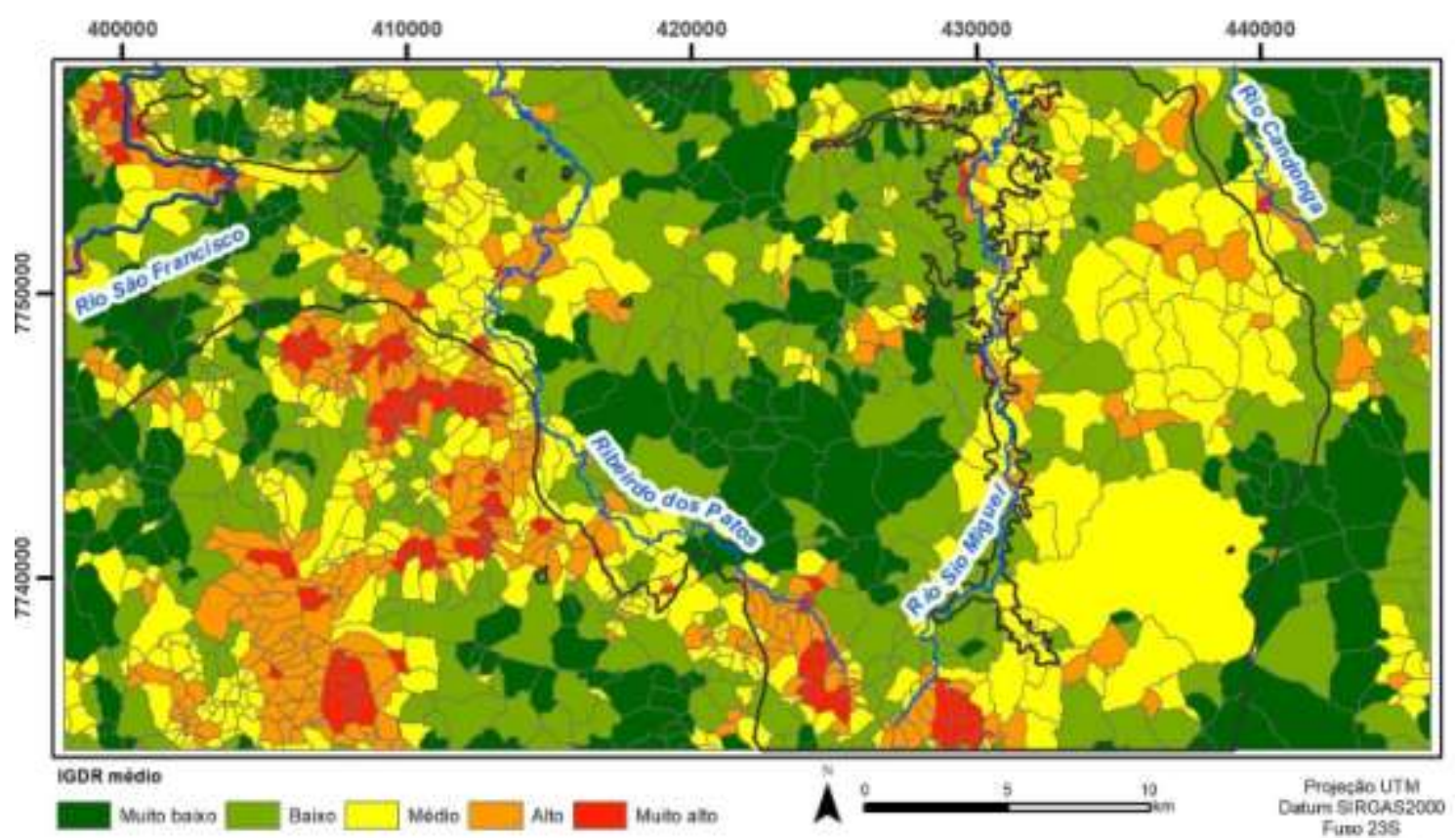

Figura 4 - Média do Índice Global de Dissecação do relevo das sub-bacias da Região Cárstica de ArcosPains-Doresópolis-Iguatama 
As Figuras 5 e 6 correspondem, respectivamente, as plotagens do Y predito e dosresíduos, sendo possível identificar a maior dispersão de outiliers em valores de geodiversidade acima do Y predito. Em contrapartida, os resultados da aplicação do modelo GWR demonstraram que existem melhores ajustamentos a nível local, como pode ser visualizado na Figura 7.

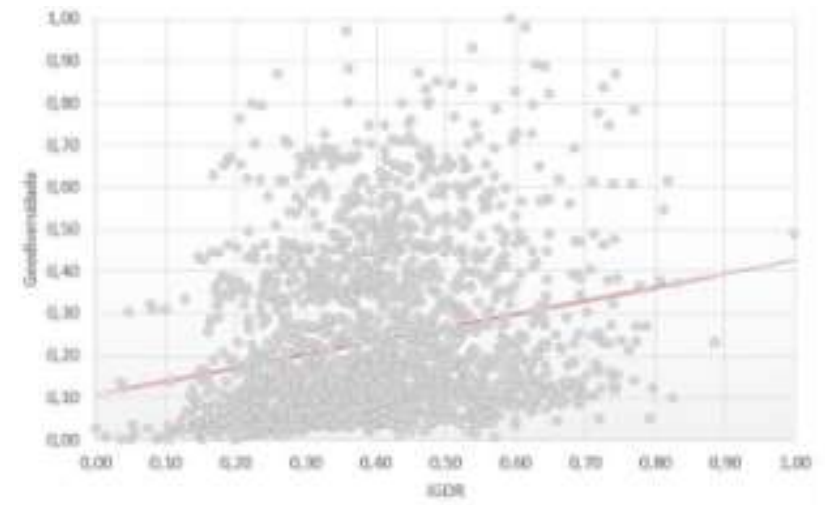

Figura 5 -Gráfico deAjuste do Y predito

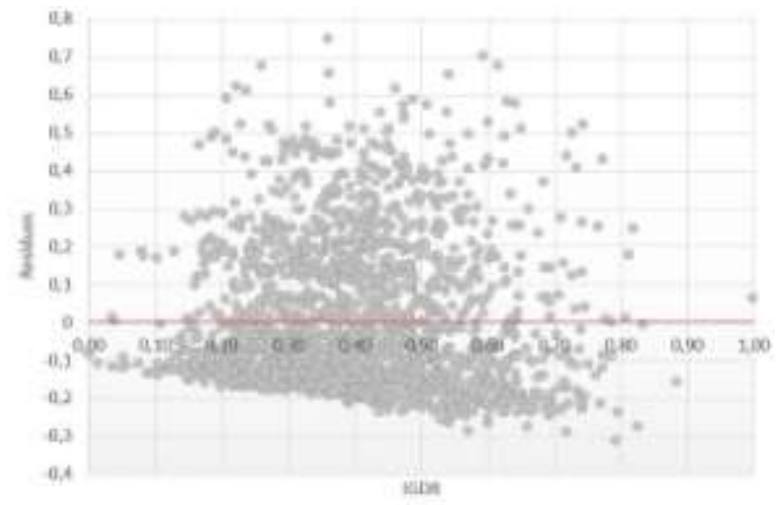

Figura 6 - Gráfico de Plotagem dos resíduos

De modo geral, pode-se inferir que a variação do IGDR influi na geodiversidade daregião, mas não consegue explicar o modelo por completo. Em apenas 5,5\% das bacias o R2 local alcançou um nível de predição satisfatório (superior a 0,5), isso significa que existem outras variáveis importantes influenciando na dinâmica da geodiversidade na grande maioria das bacias. Ao visualizar a Figura 8, que corresponde a espacialização dos escore de resíduos padronizados, podemos averiguar a discrepância dos ajustamentos e assim, analisar a efetividade do modelo.

Os resultados apontam que $73,24 \%$ das bacias possuem resíduos com desvio padrãoentre 1 a 1 , o que pode ser considerado aceitável. As bacias com geodiversidade muito superior ao ajustamento do Y predito (14,94\%), ou seja, maiores que o esperado, se encontram dispersas em ambos sistemas existentes. Supõe-se que nessas áreas estão situadas variáveis que estão inversamente relacionadas com altos valores de IGDR, como, por exemplo, deposições aluviais, dolinas e ambientes lênticos (lagoas). Além disso, a diversidade litológica influi na quantificação da geodiversidade, porém está condicionada a fatores endógenos. 


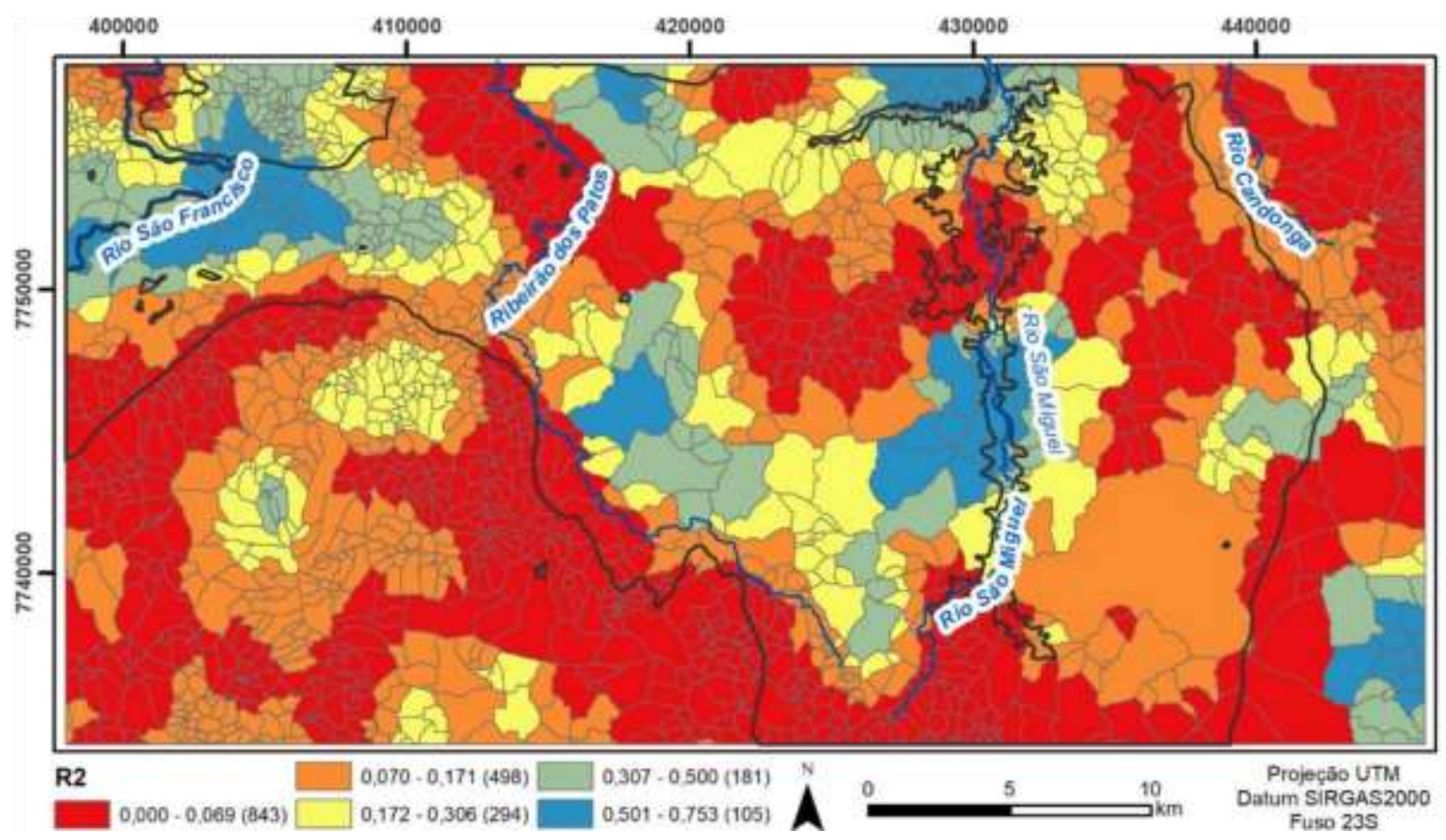

Figura 7 - Espacialização do R² Local do modelo GWR

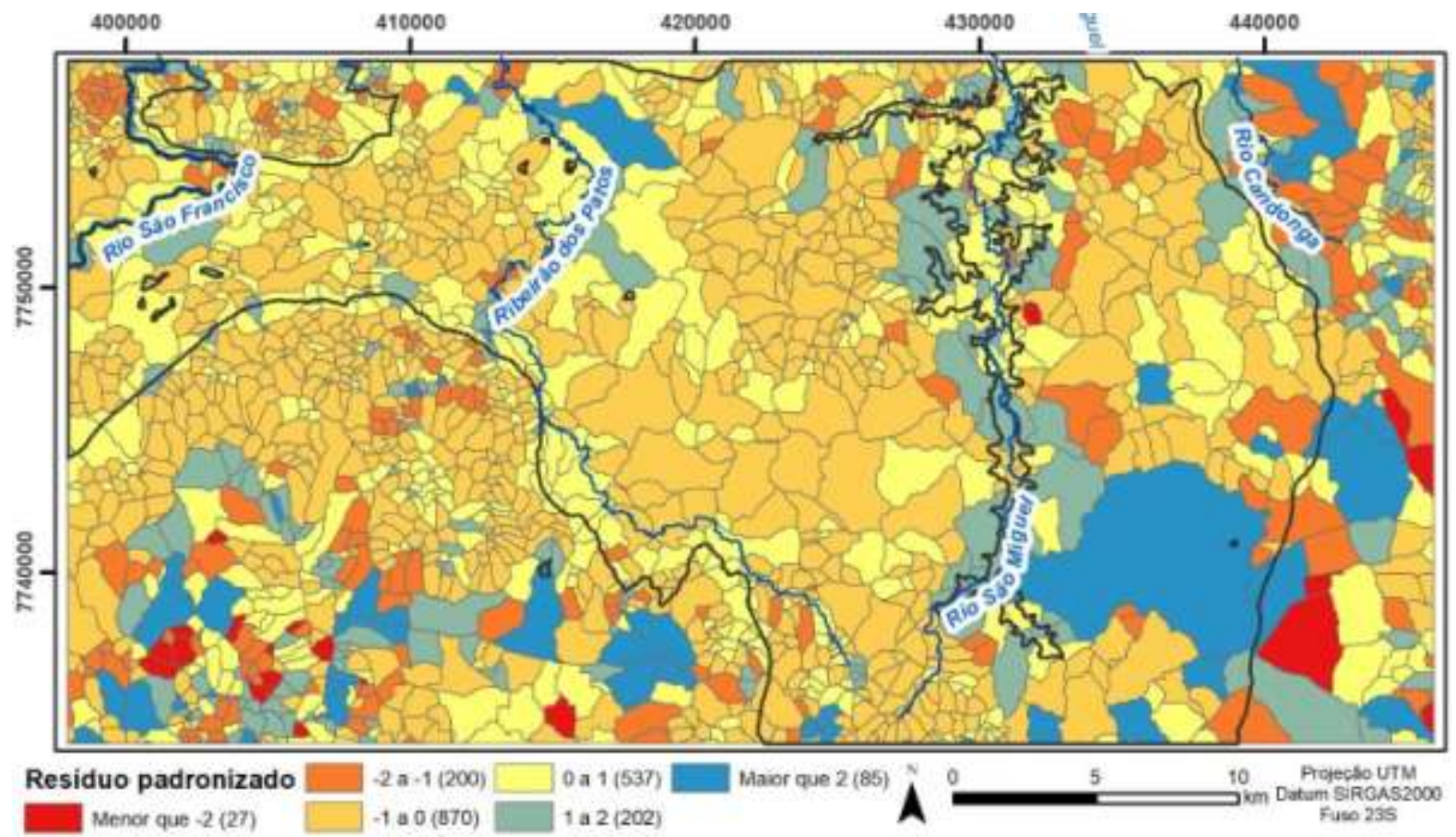

Figura 8 - Espacialização do Resíduo Padronizado do modelo GWR.

As bacias caracterizadas pelos resíduos menores que o esperado $(11,82 \%)$ seencontram em agrupamentos dispersos, onde é possível destacar as porções leste e sul/sudoeste.

Na primeira porção, as bacias, em sua grande maioria, possuem IGDR baixo e muito baixo. 
Levando em consideração o resultado do modelo, supõe-se que estas áreas possuem geodiversidade praticamente insignificante. Por outro lado, as bacias da segunda porção devem ser analisadas isoladamente, já que seus valores de IGDR são consideráveis.

\section{6- CONSIDERAÇÕES FINAIS}

Ficou evidente neste estudo as vantagens da utilização do modelo GeographicallyWeightedRegression em análises espaciais, sobretudo quando aplicadas em unidades espaciais bem definidas. Os dados gerados permitiram análises isoladas, bem como a identificação de comportamentos locais semelhantes, que resultaram em agrupamentos regionais.

Em relação a hipótese aqui levantada, vimos que o Índice Global de Dissecação do Relevo não consegue explicar sozinho o comportamento da geodiversidade. Pode-se inferir que na maioria das bacias existem variáveis mais importantes interferindo na relação. No entanto, é válido o emprego do IGDR em pesquisas de geodiversidade voltadas para ambientes tropicais tradicionais, uma vez que o índice indica processos de dissecação por erosão pluvial, levando em consideração o volume e a energia cinética da água.

Uma vez que a área de estudo é composta em sua maioria por ambientes cársticos,foram consideradas no modelo feições do exocarste e cavidades naturais, que, por sua vez, estão intrinsecamente ligadas a estrutura e composição das rochas carbonáticas. Além disso, o processo de carstificação depende de situações inversamente proporcionais à suposição deste artigo: dependência de relevos suavizados para o acúmulo e infiltração da água, como explica Pellitero (2012). Microfeições do exocarste podem ser encontrados em relevo acidentado, no entanto, estão associados aos afloramentos, já inseridos no cálculo. Neste caso, a inserção dessas variáveis poderia supervalorizar as regiões de afloramento.

É importante enfatizar que esta metodologia de quantificação pode omitir ageodiversidade em ambientes cársticos, por equiparar cavidades de diferentes graus de relevância e não considerar a geodiversidade do endocarste. Portanto, a utilização desta metodologia não exclui a necessidade de análises qualitativas e trabalhos de campo.

Concluindo, a variável rugosidade atua na equação como um fator de “causa”,proporcionando alta geodiversidade onde ocorre maior variação do relevo. No entanto, pode gerar efeitos de supervalorização ou subvalorização em determinadas regiões, o que provavelmente aconteceria se fosse utilizado nessa pesquisa. Do ponto de vista prático, pode se dizer que a utilização do coeficiente é válido em metodologias indiretas (sem utilização de variáveis 
já mapeadas) e em pesquisas exploratórias, para quantificar regiões desconhecidas, principalmente em escalas de menor detalhamento.

\section{REFERÊNCIAS BIBLIOGRÁFICAS}

BENITO-CALVO, Afonso; PÉREZ-GONZÁLEZ, A; MAGRI, O. Assessing regional geodiversity: theIberianPeninsula. Earth Surface Processes andLandforms, 34:1433 - 1445, 2009.

CASSETI, Valter. Geomorfologia. $2005 . \quad$ Disponível em: <http://www.funape.org.br/geomorfologia/\&gt>. Acesso em: nov. 2017.

CHRISTOFOLETTI, Antônio. Modelagem de Sistemas Ambientais. 1ª ed. São Paulo: Edgard Blücher, 1999.

GRAY, Murray. Geodiversity: valuing and conserving abiotic nature. John Wiley \& Sons, Chichester, England, 448 p, 2004.

HACK, J. T. Stream-profile analysis and stream-gradient index. Journal of Research of the United States Geological Survey, v. 1, n. 4, p. 421-429, 1973.

HJORT, Jan; LUOTO, Miska.Geodiversity of high-latitude landscapes in northern Finland. Elsevier Geomorphology, Finlândia, p.109-116, fev., 2010.

HOBSON, R. D.: Surface roughness in topography: a quantitative approach, Harper and Row, Methuer, Londres, p. 221-245, 1972.

HORTON, R.E. Erosional development of streams and their drainage basins: hydrophysical approach to quantitative morphology. BulletinoftheGeologicalSocietyofAmerica Bull. n.56, 1945.

LOBO, Carlos; FONSECA, Diego Ferreira, GARCIA, Ricardo Alexandrino. Verticalização e permeabilização do solo urbano nos setores censitários de Belo Horizonte/MG. Revista brasileira de estudos urbanos e regionais. Recife, v.17, p. 215-228, dez. 2015.

MARTINS, Thallita. Mapeamento Geomorfológico da Folha Piumhi, MG. 2013. 157 f. Dissertação (Mestrado em Geografia) - Programa de Pós-Graduação em Geografia, Instituto de Geografia, Universidade Federal de Uberlândia, Uberlândia, 2013.

PELLITERO, Ramón Ondicol. Geomorfología, paleoambientecuaternario y geodiversidadenelMacizo de Fuentes Carrionas-MontañaPalentina. Tese de Doutorado, Departamento de Geografia, Universidade de Valladolid, p. 1085, 2012.

PEREIRA, Eric Oliveira; RUCHKYS, Úrsula; PELLITERO, Ramón Ondicol. Modelagem da Geodiversidade na Área de Proteção Ambiental Sul da Região Metropolitana de Belo Horizonte MG. Geonomos, v. 21, p. 97-101, 2013.

SAMPAIO, T.V.M. Parâmetros morfométricos para melhoria da acurácia do mapeamento da rede de Drenagem uma proposta baseada na análise da Bacia Hidrográfica do Rio Benevente -ES. Tese de Doutorado. IGC/UFMG.Belo Horizonte, 2008. 
SERRANO, Enrique; RUIZ FLAÑO, Purificacíon. Geodiversidad: Concepto, Evaluación y Aplicación Territorial. El Caso De Tiermes (Caracena). Boletín de la A.G.E. n. 45, p. 79-98, 2007.

SOCIEDADE EXCURSIONISTA ESPELEOLÓGICA -SEE; Departamento de Geologia da Escola de Minas; Universidade Federal de Ouro Preto. PROJETO ARCOS PAINS ESPELEOLOGIA - PROAPE. Ouro Preto, 2012.

SOUZA, Fabiano Érico; FONSEA, Bráulio; COELHO, Fernanda. Compartimentação do relevo baseada em parâmetros morfométricos: uma proposta de índice global de dissecação do relevo. I congresso nacional de geografia física, Campinas, v.1, p. 1-12, jul. 2017. 


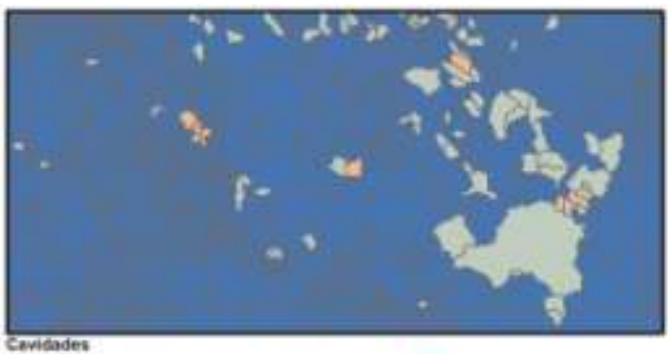

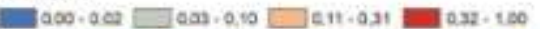

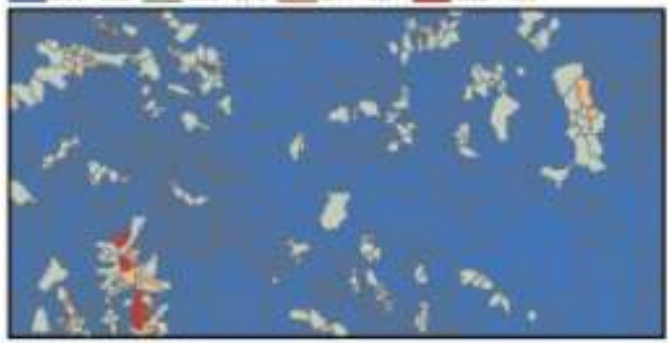

Exirutuasasinneamentes

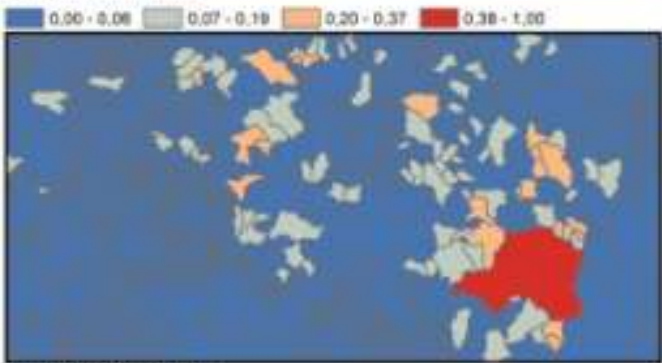

Stamifoumos murgenereis:

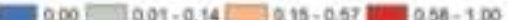

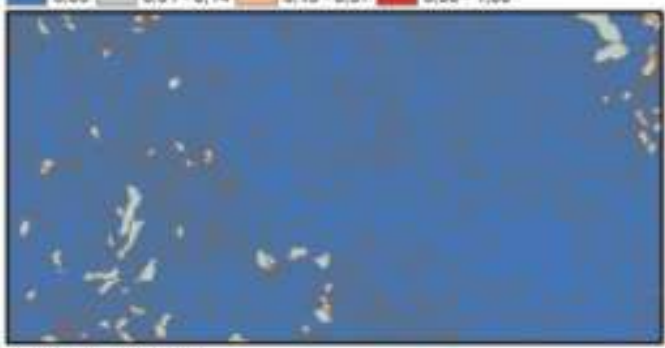

Somideurosiourgent

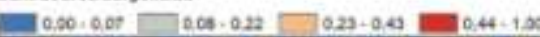

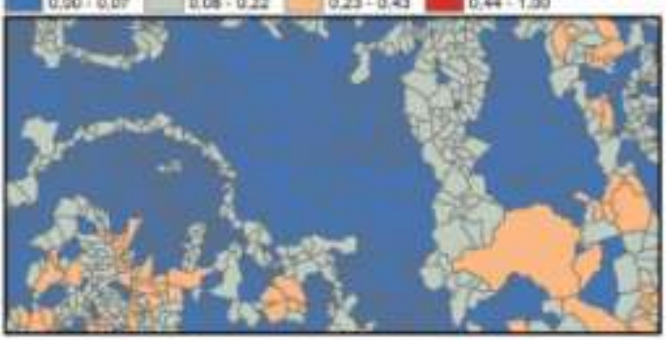

LRologia 불 $0.00 \square 0.01-0.33 \square 0.34-0.67 \square 0.68-1.00$

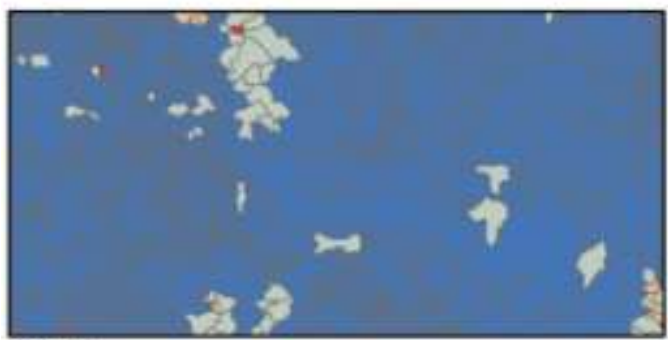

Feiçōes

$=0.00-0.06=0.07-0.22 \pm 0.23-0.53=0.54-1.00$

Cadernos do Leste

Artigos Cientificos

\section{Anexo}

Variáveis utilizadas no Índice de Geodiversidade

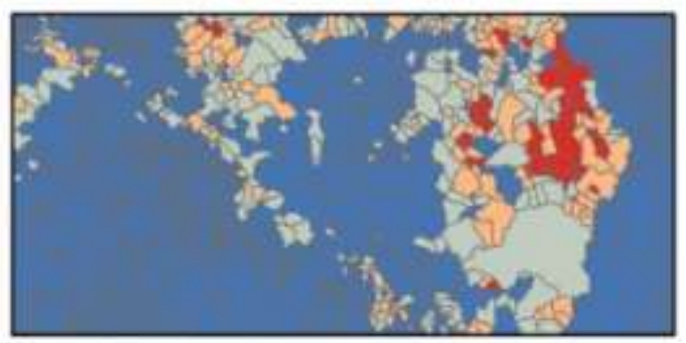

Afloramentos carsle

$=0.00-0.05 \square 0.06 \cdot 0,18 \boxminus 0,19 \cdot 0,40=0,41+1,00$

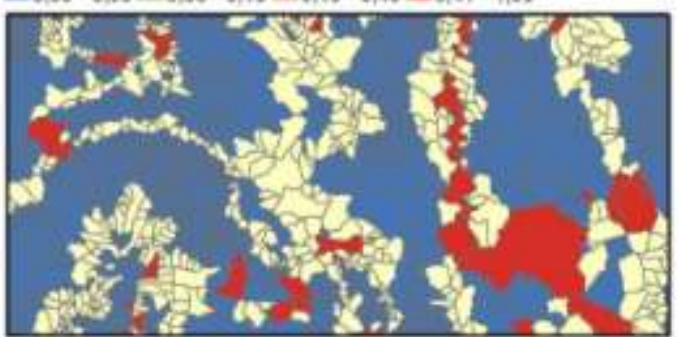

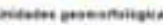

$=0,00=0.01 \cdot 0.50=0.51-1.00$

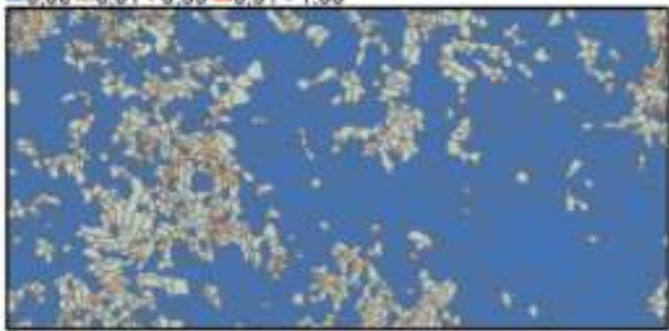

Drenagem

$=0,00-0,14 \square 0,15 \cdot 0,26 \square 0,27-0,46=0,47-1,00$

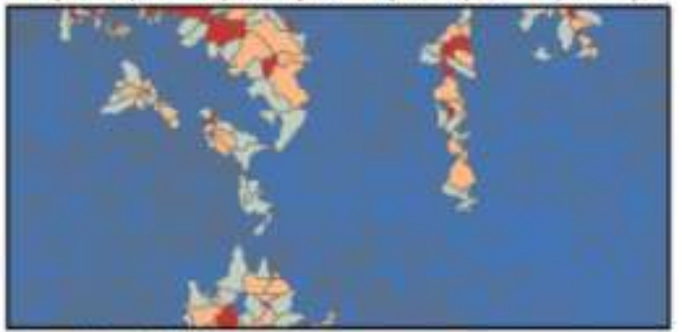

Deposiçóes aluviais

$=0.00-0.10 \square 0.11-0.34 \square 0.35-0.67=0.68-1.00$

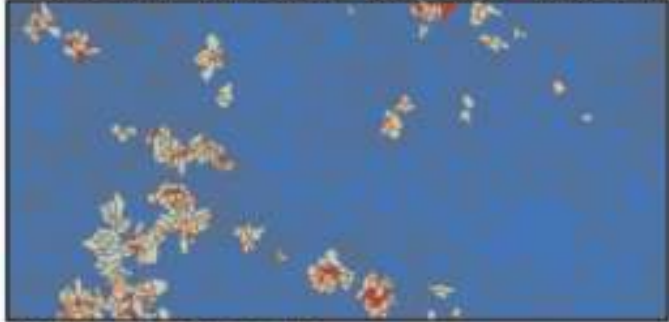

Processos erosivos recentes

$=0.00 \cdot 0.09=0.10 \cdot 0.28=0.29 \cdot 0.50=0.51 \cdot 1.00$

Belo Horizonte, Jan.-Dez. Vol.17, n¹7, 2017 